\title{
ESTILO DE VIDA E FATORES ASSOCIADOS EM ESTUDANTES UNIVERSITÁRIOS DE EDUCAÇÃO FÍSICA
}

\author{
LIFESTYLE AND ASSOCIATED FACTORS IN PHYSICAL EDUCATION UNDERGRADUATE STUDENTS
}

\author{
Leandro Martinez Vargas ${ }^{a^{*}}$, Paulo Eduardo Redkva ${ }^{b^{*}}$, José Roberto Herrera Cantorani ${ }^{*}$, \\ Gustavo Luis Gutierrez ${ }^{\mathrm{d} * *}$ \\ aleandro.vargas@uol.com.br, bpauloredkva@hotmail.com, ccantorani@yahoo.com.br, ${ }^{\mathrm{b}}$ gutierrez@fef.unicamp.br \\ *Faculdades Integradas de Itararé - Itararé (SP), Brasil \\ ${ }^{* *}$ Faculdade de Educação Física da Universidade Estadual de Campinas - Campinas (SP), Brasil
}

Data de entrada do artigo: 17/04/2014

Data de aceite do artigo: 15/08/2014

\section{RESUMO}

Introduçáo: Padróes de comportamento que compôem as esferas do estilo de vida e sua relação com fatores independentes têm sido motivo de estudo de diversos pesquisadores da área da saúde. Os estudantes universitários são, particularmente, alvo constante desse tipo de pesquisa por configurarem-se como um grupo em que há um aumento dos índices dos hábitos considerados de risco. Objetivo: Verificar a classificação global do estilo de vida e a relação com os domínios do estilo de vida em acadêmicos de licenciatura em Educaçáo Física da modalidade à distância de uma universidade pública do estado do Paraná. Materiais e Métodos: A amostra foi composta por 204 estudantes de ambos os gêneros (50,8\% do gênero masculino) com idade média de $33,5 \pm 8,3$. Foram aplicados questionários para obter dados sobre as condiçóes demográficas, socioeconômicas e sobre seus estilos de vida. Análises de independência através do teste Qui-quadrado foram realizadas para verificar a dependência entre as variáveis. Resultados: Pelos resultados obtidos da análise de dependência entre as variáveis é possível perceber que as áreas que mais impactaram $(\mathrm{p}<0,05)$ sobre o estilo de vida dos estudantes foram as relacionadas aos aspectos afetivo (família), psicológico ou emocional (estresse, comportamento/satisfação) e trabalho. Conclusóes: A socialização, a dieta balanceada, dormir bem, controlar o estresse do dia-a-dia, relaxar e desfrutar do tempo livre, controlar a raiva e a tensão, e pensar de forma positiva e otimista são alguns dos fatores que podem estar contribuindo para a maior probabilidade de possuir um estilo de vida adequado entre os universitários.

Palavras-chave: Estilo de vida; estudantes; fatores associados; educação a distância.

\section{ABSTRACT}

Introduction: Patterns of behavior that constitutes the spheres of lifestyle and its relationship with independent factors has been the subject of study of many researchers in the field of health. College students are particularly constant targets by this kind of research because they are configured as a group that there is an increased rate of the risk factors. Objective: Verify the global rating of lifestyle and relationship with the domains of lifestyle on Physical Education bachelor students of the distance learning education from a public university in the state of Paraná. Methods: The sample consisted of 204 students of both genders (50.8\% males) with a mean age of $33.5 \pm 8.3$. Questionnaires were used to obtain data on demographic and socioeconomic conditions, and also lifestyle. Independence was analyzed using Chi-square test, performed to investigate the dependence between the variables. Results: By the results obtained from the analysis of dependence between variables it was possible to see that the areas most affected $(\mathrm{p}<0.05)$ on the lifestyle of the students were related to affective aspects (family), psychological, or emotional (stress, behavior/satisfaction), and work. Conclusions: Socialization, a balanced diet, sleeping well, manage the 
stress of everyday life, relax and enjoy the free time and think positively and optimistically are some of the factors that may be contributing to the greater likelihood of a proper lifestyle among university.

Keywords: Lifestyle; students; associated factors; distance education.

\section{Introdução}

O estilo de vida pode ser considerado como o resultado dos padrōes de comportamento que o indivíduo desenvolve no decorrer da sua vida, os quais são determinados por diversos fatores, sendo estes de características pessoais, socioeconômicas e ambientais e, por consequência, acarretam um efeito profundo na saúde dos indivíduos ${ }^{1,2}$.

Segundo Rozmus et al. ${ }^{3}$, o período de ingresso no ensino superior representa uma fase importante da vida das pessoas, pois muitos comportamentos que foram adquiridos durante a vida podem ser alterados decorrentes de novas amizades, atitudes e conhecimentos. Quando se trata de um curso superior de Educação Física, o estilo de vida é um desses comportamentos que podem ser alterados com o ingresso na universidade.

$\mathrm{O}$ estilo de vida tem sido motivo de estudo de diversos pesquisadores da área da saúde e da psicologia por ser um dos mais importantes determinantes de saúde da população. Entretanto, apesar de toda informação a respeito do tema, as pesquisas comportamentais revelam que entre os estudantes universitários há um aumento dos índices e da quantidade de hábitos considerados de risco ${ }^{4}$.

Grande parte da comunidade científica específica defende que a identificaçáo dos comportamentos de risco poderia contribuir para a prevenção - ou adiamento - de algumas enfermidades. Entretanto, segundo Palma, Abreu e Cunha ${ }^{5}$, a noção de comportamento de risco estimula um envolvimento individual mais ativo com a prevençáo e imputa ao próprio ser a responsabilidade por seu adoecimento. Assim, a opçáo de aceitabilidade do risco, portanto, implicaria ao próprio indivíduo uma condição de autorresponsabilidade pela decisão de abrandá-lo ou, se possível, eliminá-lo. Segundo os autores, as noçóes de "comportamento de risco" ou de seu antônimo, cunhado pragmaticamente pelas ciências positivistas como "estilo de vida saudável" se inserem neste contexto.

A literatura traz diversos estudos que revelam o perfil do estilo de vida de acadêmicos de diversos cursos na modalidade presencial ${ }^{1,4,9}$, sendo alguns especificamente com alunos de Educaçáo Física ${ }^{10,12}$. Os resultados revelam que esse grupo de universitários apresenta melhores níveis de estilo de vida, qualidade de vida e atividade física quando comparados a acadêmicos de outras áreas, inclusive da saúde ${ }^{9,10}$.
Logo, o que instiga o presente estudo diz respeito ao fato de conhecer se os futuros professores de Educação Física conduzem seu estilo de vida através de comportamentos considerados saudáveis, que condizem com os conhecimentos adquiridos ao longo do curso e com o discurso que representam. Além do mais, em nossa pesquisa, nenhum estudo publicado na literatura científica se preocupou em descrever o estilo de vida dos acadêmicos egressos de um curso na modalidade à distância.

Nesse contexto, conhecer os fatores relacionados ao estilo de vida adequado pode subsidiar a realização de políticas e programas de intervenção direcionados a promoção da saúde dessa particular população de universitários, que, por um lado, é a que mais cresce no Brasil, mas, em contra partida, ainda carente de estudos epidemiológicos e comportamentais.. Assim, a presente pesquisa tem por finalidade verificar a classificação global do estilo de vida e a relação com os domínios do estilo de vida em acadêmicos de licenciatura em Educação Física da modalidade à distância de uma universidade pública do estado do Paraná.

\section{Metodologia}

Trata-se de uma pesquisa descritiva de corte transversal com abordagem quantitativa, na qual a população objeto de estudo foi composta por acadêmicos de ambos os gêneros, regularmente matriculados no curso de licenciatura em Educaçáo Física a distância da Universidade Estadual de Ponta Grossa oriundos das cidades polo de Apucarana, Bituruna, Congonhinhas, Cruzeiro do Oeste, Ibaiti, Lapa, Palmeira, Paranaguá e Siqueira Campos, todas localizadas no interior do estado do Paraná.

Para determinaçáo do tamanho amostral foi realizada técnica de amostragem estratificada com correção para populaçóes finitas. Para o cálculo amostral considerou-se o valor estimado do desfecho estudado alunos com estilo de vida adequado - igual a 50\% (p $=0,50)$. Optou-se, ainda, por um nível de confiança de $99 \%$ e por uma precisão de 5 pontos percentuais. Assim, o número de indivíduos necessários para compor a amostra foi de 213 estudantes universitários. A seleção da amostra foi realizada pelo método aleatório simples, através de sorteio dos registros acadêmicos dos estudantes. 
A coleta de dados ocorreu no final no mês de junho de 2013 e procedeu-se a partir de dois questionários eletrônicos autoaplicáveis ${ }^{13}$. Cada questionário possuía uma descrição esclarecendo as questóes, instruindo os acadêmicos respondentes a cada etapa.

No primeiro questionário foram levantadas as variáveis demográficas-socioeconômicas: gênero (masculino e feminino), idade (<30 anos; $\geq 30$ anos), peso (quilos), estatura (metros), situação conjugal (solteiro/ divorciado/viúvo; casado/união estável), nível econômico (A, B, C, D ou E) e grau de escolaridade (grau incompleto; grau completo). O nível socioeconômico foi avaliado por meio do Critério de Classificação Econômica Brasil, baseado no levantamento de bens de consumo, escolaridade do chefe de família e a presença de empregada doméstica ${ }^{14}$. Devido à necessidade de distribuir as variáveis em frequências, decidiu-se unir as classes "A" e "B" e denominá-la de "alta" e, as demais, de "baixa". Essa distribuição pode ser encontrada em outros estudos ${ }^{12}$. Incluiu-se ainda a autopercepção de saúde, que é considerada um indicador válido e relevante do estado de saúde de indivíduos e populaçóes ${ }^{15}$. As opçóes de resposta foram: Insatisfeito e Satisfeito.

O segundo questionário, denominado "Estilo de vida fantástico", traduzido e validado no Brasil por Añes, Reis e Petroski ${ }^{16}$, teve por objetivo mensurar os principais elementos que caracterizam o estilo de vida adequado para a saúde. Desenvolvido no Departamento de Medicina Familiar da Universidade McMaster, no Canadá, por Wilson e Ciliska ${ }^{17}$, o instrumento é composto por 25 questóes fechadas que exploram nove domínios: 1) família e amigos; 2) atividade física; 3) nutrição; 4) cigarro e drogas; 5) álcool; 6) sono, cinto de segurança, estresse e sexo seguro; 7) tipo de comportamento; 8) introspecção; 9) trabalho.

As questões, auto respondidas, consideram o comportamento dos indivíduos no último mês e os resultados permitem associar o estilo de vida e a saúde. A soma dos escores de cada domínio é classificada em cinco categorias, porém no presente estudo as mesmas foram dicotomizadas, considerando como "Adequado" os sujeitos das categorias "Excelente", "Muito bom" e "Bom", e como "Inadequado" os das categorias "Regular" e "Necessita melhorar".

O tratamento estatístico foi realizado por meio do programa Statistical Package for the Social Sciences (SPSS), versão 20. Foi utilizada a estatística descritiva e inferencial. Para verificar a normalidade dos dados foi efetuado o teste de Kolmogorov-Smirnov, indicando que tais dados seguem uma distribuiçáo normal, permitindo, assim, a avaliação por meio da estatística paramétrica. Foi feita análise descritiva das variáveis do estudo e os resultados expressos em tabelas de frequência. Em seguida, foram realizadas análises de independência entre variáveis através do teste Qui-quadrado e teste exato de Fischer para heterogeneidade e tendência linear. Todas as associaçóes que apresentaram um $\mathrm{p} \leq 0,05$ na análise de independência entre variáveis foram consideradas significantes ${ }^{18}$.

Baseado nas preocupaçóes éticas e metodológicas discutidas nas Diretrizes e Normas Regulamentadoras das Pesquisas Envolvendo Seres Humanos (Resolução $466 / 2012$ ), o presente estudo teve o seu projeto de pesquisa encaminhado à Comissão de Ética em Pesquisa da Universidade Estadual de Ponta Grossa (COEP-UEPG), sendo aprovado sob o parecer $n^{\circ}$ $451.209 / 2013$.

\section{Resultados}

No processo, dos 213 estudantes selecionados, 204 aceitaram participar da pesquisa. Desses, 15 foram considerados perdas amostrais por não terem preenchido por completo os questionários. Assim, 189 acadêmicos do curso de Licenciatura em Educação Física a distância da Universidade Estadual de Ponta Grossa responderam aos questionários de forma completa, apresentando taxa de resposta de 92,6\%. Na Tabela 1, observa-se as características demográficas e socioeconômicas dos acadêmicos.

A Figura 1 descreve a distribuição dos acadêmicos em relação ao estilo de vida. Dos universitários analisados, somente 4,2\% (IC95\%: 1,5-6,9) apresentou um estilo de vida inadequado. Em relação aos domínios do estilo de vida, foi observado que 5,8\% (IC95\%: 2,6-9,0) apresentaram uma relação inadequada com a família/amigos, 50,8\% (IC95\%: 43,9-57,6) não atenderam as recomendaçôes para atividade física, 27,5\% (IC95\%: 21,4-33,6) declararam uma alimentação inadequada, 0,5\% (IC95\%: 0,04-1,4) faziam uso de tabaco e/ou outros tóxicos, 1,6\% (IC95\%: 1,2-3,3) ingeriram bebidas alcoólicas em excesso, 4,8\% (IC95\%:1,8-7,7) reportaram problemas com sono, estresse e comportamentos de risco ao dirigir e praticar sexo, 29,1\% (IC95\%:22,8-35) sentiam-se frequentemente com raiva e hostis, 29,1\% (IC95\%: 22,8-35,3) apresentavam problemas de introspecção, e apenas $0,5 \%$ (IC95\%:0,04-1,4) se mostraram insatisfeitos com o trabalho que exerciam.

Entre as questôes correspondentes aos domínios, as que se correlacionaram com o estilo de vida foram: ter alguém para conversar sobre coisas que são importantes, dar e receber afeto, ter uma dieta balanceada, dormir bem e se sentir descansado, ser capaz de lidar com o estresse no dia-a-dia, relaxar e desfrutar do tempo livre, não sentir-se com raiva e hostil, pensar de forma positiva e otimista, não sentir-se tenso e desapontado e estar satisfeito com o trabalho $(\mathrm{p} \leq 0,05)$ (Tabela 2). 
Tabela 1: Características demográficas e socioeconômicas dos estudantes de licenciatura em Educação Física a distância. Paraná. $(\mathrm{n}=189)$

\begin{tabular}{|c|c|c|c|c|}
\hline Sexo & $\mathbf{n}$ & $\%$ & \multicolumn{2}{|c|}{ IC95\% } \\
\hline & & & & \\
\hline Homens & 96 & 50,8 & 44,0 & 57,6 \\
\hline Mulheres & 93 & 49,2 & 42,4 & 56,0 \\
\hline \multicolumn{5}{|l|}{ Trabalho remunerado } \\
\hline$<\mathrm{ou}=8$ horas diárias & 139 & 73,5 & 66,7 & 80,4 \\
\hline$>8$ horas diárias & 50 & 26,5 & 19,6 & 33,3 \\
\hline \multicolumn{5}{|l|}{ Faixa etária (anos) } \\
\hline $18-29$ & 65 & 34,4 & 27,5 & 41,2 \\
\hline $30-39$ & 82 & 43,4 & 36,5 & 50,2 \\
\hline $40-49$ & 33 & 17,5 & 10,6 & 24,3 \\
\hline $50-59$ & 8 & 4,2 & 2,6 & 11,1 \\
\hline $60-69$ & 1 & 0,5 & 6,3 & 7,4 \\
\hline \multicolumn{5}{|c|}{ Classe econômica - Renda média familiar } \\
\hline \multicolumn{5}{|c|}{ (Valor bruto em R\$) * } \\
\hline A2 $(11.965,00)$ & 19 & 10,1 & 3,2 & 16,9 \\
\hline B1 $(6.280,00)$ & 60 & 31,7 & 24,9 & 38,6 \\
\hline B2 $(3.646,00)$ & 87 & 46,0 & 39,2 & 52,9 \\
\hline C1 $(2.190,00)$ & 23 & 12,2 & 5,3 & 19,0 \\
\hline C2 $(1.455,00)$ & 1 & 0,5 & 6,3 & 7,4 \\
\hline \multicolumn{5}{|l|}{ Situaçáo conjugal } \\
\hline Casado legalmente & 101 & 53,4 & 46,6 & 60,3 \\
\hline Separado/Divorciado & 9 & 4,8 & 2,1 & 11,6 \\
\hline União estável & 24 & 12,7 & 5,9 & 19,5 \\
\hline Solteiro & 55 & 29,1 & 22,3 & 35,9 \\
\hline \multicolumn{5}{|l|}{ Escolaridade } \\
\hline $3^{\circ}$ Grau Incompleto & 129 & 68,3 & 61,4 & 75,1 \\
\hline $3^{\circ}$ Grau Completo e Pós-graduação & 60 & 31,7 & 24,9 & 38,6 \\
\hline \multicolumn{5}{|l|}{ Índice de massa corporal $(\mathrm{kg} / \mathrm{m} 2)$} \\
\hline \multicolumn{5}{|l|}{ Homens } \\
\hline Abaixo do peso $(<19,9)$ & 3 & 3,1 & 3,7 & 10,0 \\
\hline Peso normal $(21,0-24,9)$ & 28 & 29,2 & 22,3 & 36,0 \\
\hline Acima do peso $(>24,9)$ & 65 & 67,7 & 60,9 & 74,6 \\
\hline \multicolumn{5}{|l|}{ Mulheres } \\
\hline Abaixo do peso $(<19,9)$ & 8 & 8,6 & 1,8 & 15,4 \\
\hline
\end{tabular}

Pelos resultados obtidos da análise de dependência entre as variáveis é possível perceber que as áreas que mais impactaram $(\mathrm{p}<0,05)$ sobre o estilo de vida dos estudantes foram as relacionadas aos aspectos afetivo (família), psicológico ou emocional (estresse, comportamento/satisfação) e trabalho.

\section{Discussão}

Em relação ao estilo de vida, 95,7\% da amostra recebeu a classificação adequada. A maior parte dos universitários investigados eram homens com mais de 30 anos de idade, casados ou com união estável, cursavam pela primeira vez um curso superior, trabalham de forma remunerada oito ou mais horas por dia, de nível econômico alto e IMC acima de $25 \mathrm{~kg} / \mathrm{m}^{2}$.

As questóes relacionadas ao domínio família e amigos, "tenho alguém para conversar as coisas que são importantes para mim" e "dou e recebo afeto" mostraram-se estar fortemente associadas com um estilo de vida global adequado. A nova rede de amizades criadas no âmbito universitário, culminado em um novo processo de socialização diferenciado do até então, pode ter contribuído para o elevado índice de acadêmicos que possuem alguém para conversar e dar e receber a feto.

Nahas ${ }^{19}$ traz que o relacionamento consigo mesmo e com pessoas a sua volta representa um dos componentes 
Tabela 2: Resultados da análise de dependência entre o Estilo de Vida Global e as variáveis do Estilo de Vida. Paraná, Brasil, 2013

\begin{tabular}{|c|c|c|c|c|c|c|c|}
\hline \multirow{2}{*}{ Variável } & \multicolumn{2}{|c|}{ Adequado } & \multicolumn{2}{|c|}{ Inadequado } & \multicolumn{2}{|c|}{ Total } & \multirow{2}{*}{ valor-1 } \\
\hline & $\mathbf{n}$ & $\%$ & $\mathbf{n}$ & $\%$ & $\mathbf{n}$ & $\%$ & \\
\hline \multirow{2}{*}{\multicolumn{8}{|c|}{$\begin{array}{l}\text { Tenho alguém para conversar as coisas que sáo } \\
\text { importantes para mim }\end{array}$}} \\
\hline & & & & & & & \\
\hline Adequado & 176 & 97,8 & 4 & 2,2 & 180 & 95,2 & \multirow{2}{*}{0,000} \\
\hline Inadequado & 5 & 55,6 & 4 & 44,4 & 9 & 4,8 & \\
\hline \multicolumn{8}{|c|}{ Dou e recebo afeto } \\
\hline Adequado & 174 & 97,8 & 4 & 2,2 & 178 & 94,2 & \multirow{2}{*}{0,000} \\
\hline Inadequado & 7 & 63,6 & 4 & 36,4 & 11 & 5,8 & \\
\hline \multirow{3}{*}{\multicolumn{8}{|c|}{$\begin{array}{l}\text { Sou vigorosamente ativo pelo menos } \\
\text { durante } 30 \text { minutos por dia (corrida, } \\
\text { bicicleta, trabalho de casa) }\end{array}$}} \\
\hline & & & & & & & \\
\hline & & & & & & & \\
\hline Adequado & 83 & 94,3 & 5 & 5,7 & 88 & 46,6 & \multirow{2}{*}{0,287} \\
\hline Inadequado & 98 & 97,0 & 3 & 3,0 & 101 & 53,4 & \\
\hline \multicolumn{8}{|c|}{$\begin{array}{l}\text { Sou moderadamente ativo (jardinagem, } \\
\text { caminhada, trabalho de casa) }\end{array}$} \\
\hline Adequado & 93 & 97,9 & 2 & 2,1 & 95 & 50,3 & \multirow{2}{*}{0,169} \\
\hline Inadequado & 88 & 93,6 & 6 & 6,4 & 94 & 49,7 & \\
\hline \multicolumn{8}{|c|}{ Como uma dieta balanceada } \\
\hline Adequado & 140 & 98,6 & 2 & 1,4 & 142 & 75,1 & \multirow{2}{*}{0,003} \\
\hline Inadequado & 41 & 87,2 & 6 & 12,8 & 47 & 24,9 & \\
\hline \multicolumn{8}{|c|}{ Frequentemente como em excesso } \\
\hline \multicolumn{8}{|c|}{ (1) açúcar, (2) sal, (3) gordura animal } \\
\hline \multicolumn{8}{|c|}{ (4) bobagens e salgadinho } \\
\hline Adequado & 24 & 100,0 & 0 & 0,0 & 24 & 12,7 & \multirow{2}{*}{0,599} \\
\hline Inadequado & 157 & 95,2 & 8 & 4,8 & 165 & 87,3 & \\
\hline \multicolumn{8}{|c|}{ Estou no intervalo de 4 quilos do meu } \\
\hline \multicolumn{8}{|c|}{ peso considerado saudável } \\
\hline Adequado & 106 & 98,1 & 2 & 1,9 & 108 & 57,1 & \multirow{2}{*}{0,076} \\
\hline Inadequado & 75 & 92,6 & 6 & 7,4 & 81 & 42,9 & \\
\hline Fumo cigarr & & & & & & & \\
\hline Adequado & 173 & 96,1 & 7 & 3,9 & 180 & 95,2 & 0328 \\
\hline Inadequado & 8 & 88,9 & 1 & 11,1 & 9 & 4,8 & $0,3<0$ \\
\hline Uso drogas c & & & & & & & \\
\hline Adequado & 175 & 95,6 & 8 & 4,4 & 183 & 96,8 & 1000 \\
\hline Inadequado & 6 & 100,0 & 0 & 0,0 & 6 & 3,2 & 1,000 \\
\hline Abuso de rer & & & & & & & \\
\hline Adequado & 168 & 96,6 & 6 & 3,4 & 174 & 92,1 & 0125 \\
\hline Inadequado & 13 & 86,7 & 2 & 13,3 & 15 & 7,9 & 0,125 \\
\hline Ingiro bebid & & & & & & & \\
\hline (café, chá ou & & & & & & & \\
\hline Adequado & 37 & 97,4 & 1 & 2,6 & 38 & 20,1 & 1000 \\
\hline Inadequado & 144 & 95,4 & 7 & 4,6 & 151 & 79,9 & 1,000 \\
\hline Minha inges & & & & & & & \\
\hline de álcool é d & & & & & & & \\
\hline Adequado & 171 & 95,5 & 8 & 4,5 & 179 & 94,7 & 1000 \\
\hline Inadequado & 10 & 100,0 & 0 & 0,0 & 10 & 5,3 & 1,000 \\
\hline
\end{tabular}


Tabela 2: Continuação

\begin{tabular}{|c|c|c|c|c|c|c|c|}
\hline \multirow{2}{*}{ Variável } & \multicolumn{2}{|c|}{ Adequado } & \multicolumn{2}{|c|}{ Inadequado } & \multicolumn{2}{|c|}{ Total } & \multirow{2}{*}{ valor- $\mathrm{p}$} \\
\hline & n & $\%$ & $\mathbf{n}$ & $\%$ & $\mathbf{n}$ & $\%$ & \\
\hline \multicolumn{8}{|c|}{ Bebo mais de quatro doses em uma ocasiáo } \\
\hline Adequado & 143 & 95,3 & 7 & 4,7 & 150 & 79,4 & \multirow{2}{*}{1,000} \\
\hline Inadequado & 38 & 97,4 & 1 & 2,6 & 39 & 20,6 & \\
\hline \multicolumn{8}{|c|}{ Dirijo após beber } \\
\hline Adequado & 156 & 96,3 & 6 & 3,7 & 162 & 85,7 & \multirow{2}{*}{0,320} \\
\hline Inadequado & 25 & 92,6 & 2 & 7,4 & 27 & 14,3 & \\
\hline \multicolumn{8}{|c|}{ Durmo bem e me sinto descansado } \\
\hline Adequado & 167 & 98,2 & 3 & 1,8 & 170 & 89,9 & \multirow{2}{*}{0,000} \\
\hline Inadequado & 14 & 73,7 & 5 & 26,3 & 19 & 10,1 & \\
\hline \multicolumn{8}{|c|}{ Uso cinto de segurança } \\
\hline Adequado & 131 & 95,6 & 6 & 4,4 & 137 & 72,5 & \multirow{2}{*}{1,000} \\
\hline Inadequado & 50 & 96,2 & 2 & 3,8 & 52 & 27,5 & \\
\hline \multicolumn{8}{|c|}{ Sou capaz de lidar com o estresse do meu dia-a-dia } \\
\hline Adequado & 135 & 99,3 & 1 & 0,7 & 136 & 72,0 & \multirow{2}{*}{0,001} \\
\hline Inadequado & 46 & 86,8 & 7 & 13,2 & 53 & 28,0 & \\
\hline \multicolumn{8}{|c|}{ Relaxo e desfruto do meu tempo de lazer } \\
\hline Adequado & 110 & 100,0 & 0 & 0,0 & 110 & 58,2 & \multirow{2}{*}{0,001} \\
\hline Inadequado & 71 & 89,9 & 8 & 10,1 & 79 & 41,8 & \\
\hline \multicolumn{8}{|c|}{ Pratico sexo seguro (ver explicaçáo) } \\
\hline Adequado & 121 & 97,6 & 3 & 2,4 & 124 & 65,6 & \multirow{2}{*}{0,126} \\
\hline Inadequado & 60 & 92,3 & 5 & 7,7 & 65 & 34,4 & \\
\hline \multicolumn{8}{|c|}{ Aparento estar com pressa } \\
\hline Adequado & 49 & 100,0 & 0 & 0,0 & 49 & 25,9 & \multirow{2}{*}{0,115} \\
\hline Inadequado & 132 & 94,3 & 8 & 5,7 & 140 & 74,1 & \\
\hline \multicolumn{8}{|c|}{ Sinto-me com raiva e hostil } \\
\hline Adequado & 115 & 99,1 & 1 & 0,9 & 116 & 61,4 & \multirow{2}{*}{0,006} \\
\hline Inadequado & 66 & 90,4 & 7 & 9,6 & 73 & 38,6 & \\
\hline \multicolumn{8}{|c|}{ Penso de forma positiva e otimista } \\
\hline Adequado & 129 & 98,5 & 2 & 1,5 & 131 & 69,3 & \multirow{2}{*}{0,011} \\
\hline Inadequado & 52 & 89,7 & 6 & 10,3 & 58 & 30,7 & \\
\hline Sinto-me ten & & & & & & & \\
\hline Adequado & 104 & 99,0 & 1 & 1,0 & 105 & 55,6 & 0.023 \\
\hline Inadequado & 77 & 91,7 & 7 & 8,3 & 84 & 44,4 & $0,0<J$ \\
\hline Sinto-me tri & & & & & & & \\
\hline Adequado & 117 & 98,3 & 2 & 1,7 & 119 & 63,0 & 0.053 \\
\hline Inadequado & 64 & 91,4 & 6 & 8,6 & 70 & 37,0 & נקט, \\
\hline Estou satisfe & & & & & & & \\
\hline Adequado & 100 & 100,0 & 0 & 0,0 & 100 & 52,9 & 0.002 \\
\hline Inadequado & 81 & 91,0 & 8 & 9,0 & 89 & 47,1 & \\
\hline
\end{tabular}

* Fonte: Valores atualizados ABEP (2012); Nas classes A1 (R \$ 18.373,00), D (R \$ 1.015,00) e E (R \$ 678,00) não houveram respondentes 
Figura 1: Prevalência de inadequação do estilo de vida global e por domínios dos estudantes de licenciatura em Educação Física a distância. Paraná. (n=189)

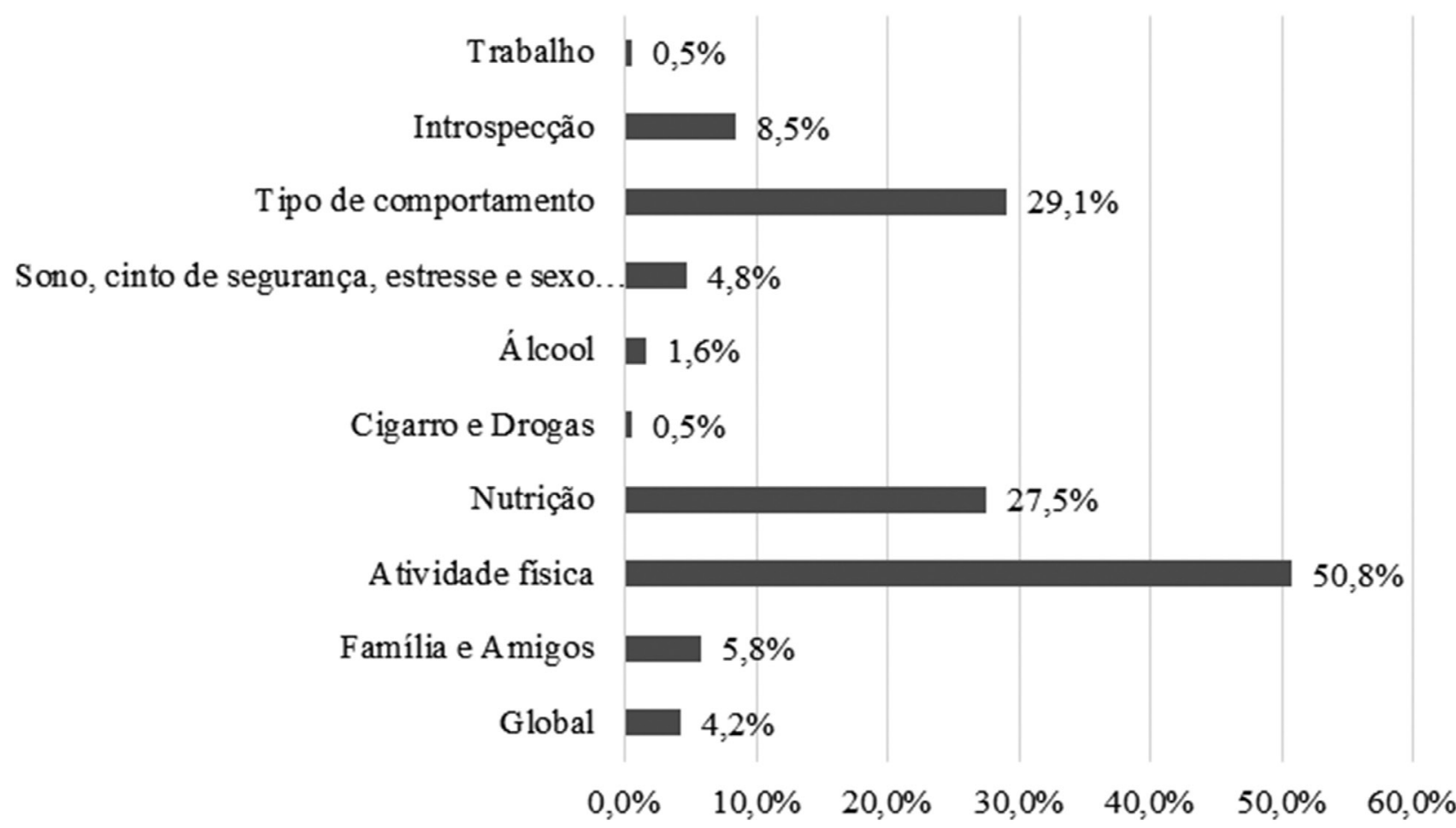

fundamentais do bem estar espiritual, o que impacta diretamente na qualidade de vida. $\mathrm{O}$ que mostra que o estudante que possui pessoas da família, ou outras pessoas também consideras importantes, as quais ela possa dividir os seus sentimentos de felicidade ou tristeza, tem maiores chances de apresentar um estilo de vida adequado.

Estudos vem mostrando que o nível de atividade física dos jovens tende a diminuir conforme o passar dos anos dentro da universidade, inclusive os matriculados em cursos da área da saúde. Em alunos de Educação Física essa tendência é menor ${ }^{7}$. No presente estudo apenas $46,6 \%$ dos estudantes afirmaram praticar pelo menos 30 minutos de atividade física vigorosa regularmente (três vezes por semana). Joia ${ }^{4}$ observou que $45,5 \%$ dos estudantes universitários entre 20 e 40 anos não praticam ao menos 30 minutos de atividade física moderada e/ou vigorosa três vezes por semana. Em Moraes et al. ${ }^{10}, 51,9 \%$ dos acadêmicos de Educação Física apresentaram índices baixos de atividade física.

A adesão à prática regular de atividade física pode ocorrer em função de três fatores: norma, gosto e uti$\operatorname{lidade}^{20}$. Sentir prazer pela atividade física e perceber a utilidade dela para a saúde e qualidade de vida pode ser o que motiva a maioria dos jovens candidatos a concorrer para um vestibular do curso de Educaçáo Física. Entretanto, há aqueles que passam a praticar atividade após a entrada na universidade, o que diz respeito à obrigatoriedade da realização da prática, ou seja, em função da norma .

Mas em relação aos estudantes classificados como sedentários, em um estudo realizado com universitários na cidade de Teresina/PI que considerou os motivos para a não realização de atividade física dos estudantes classificados como sedentários, foi possível observar que a principal razão do comportamento sedentário foi a falta de tempo $(51,7 \% ; p=0,019)^{21}$. Da mesma forma, Reichert et al. ${ }^{22}$, após entrevistarem 3.100 indivíduos, concluíram que a falta de tempo foi a barreira que se associou mais fortemente à inatividade física, indo de encontro com os estudos de Mielke et al. ${ }^{23}$ e Castro Junior et al. ${ }^{24}$ envolvendo universitários de Educação Física e da área da saúde, respectivamente.

As respostas das questóes referentes aos hábitos alimentares demonstraram certa divergência entre eles. Ao mesmo tempo em que $75,1 \%$ dos estudantes relataram manter uma dieta balanceada - que se refere a quantidade de porçóes diárias mínimas e máximas de alimentos como grãos e cereais, frutas e vegetais, derivados do leite e carnes e semelhantes $-87,3 \%$ frequentemente comem em excesso açúcar, sal, gordura animal ou bobagens e salgadinhos. Essa divergência mostra que mesmo que os estudantes controlem a ingestáo de alguns alimentos, ao exceder na ingestão diária de açúcar, sal, gordura animal 
ou bobagens e salgadinhos, podem estar colocando em risco a sua saúde.

Por mais que o estilo de vida global adequado tenha apresentado associação com a dieta balanceada ( $\mathrm{p}$-valor $=0,003)$, o que mais chama atenção é que a grande maioria dos acadêmicos frequentemente excede em alguns tipos de alimentos, o que vai de encontro a outros estudos, como os realizados com acadêmicos de Educação Física de Brasília/DF²5, de Pelotas/RS'26, São Cristovão/SE ${ }^{12}$ e Irati $/ \mathrm{PR}^{10}$. Silva ${ }^{12}$ encontrou que os acadêmicos com uma alimentação inadequada apresentaram cinco vezes mais chances de serem pouco ativos que seus pares com alimentação adequada.

As análises de dependência entre o estilo de vida global adequado e o tabagismo e o uso de bebidas alcoólicas mostraram que não houve associação estatística significativa. $\mathrm{O}$ que se observou foi um baixo índice de tabagismo e consumo de bebidas alcoólicas entre os estudantes de Educação Física a distância da UEPG $-4,8 \%$ e $5,3 \%$, respectivamente. A baixa prevalência de indivíduos que consomem álcool e fumam cigarros também foi observada em estudos com populaçôes semelhantes de outras regiôes do país, como Rio de Janeiro/RJ ${ }^{5}$, Pelotas/RS ${ }^{27}$, Juiz de Fora/ $\mathrm{MG}^{28}$ e João Pessoa/PB ${ }^{29}$. Contrapondo-se a estas observaçóes, Joia ${ }^{4}$ constatou uma alta prevalência $(53,7 \%)$ de tabagistas e etilistas entres estudantes de 10 cursos da Faculdade São Francisco de Barreiras/BA.

$\mathrm{O}$ álcool é de longe a droga mais consumida entre os jovens e um dos mais importantes fatores de risco para a adoção de outras condutas maléficas para a saúde, como o sedentarismo. No Brasil, o uso regular de bebidas alcoólicas começa aos 17 anos, ou seja, muito próximo ao ingresso à universidade ${ }^{30} \mathrm{e}$ de forma pesada, apresentando situaçóes de abuso agudo ${ }^{31}$. Entretanto, diversos outros fatores podem influenciar o consumo de bebidas alcoólicas, como por exemplo, o contexto familiar e social, poder aquisitivo, facilidade de acesso, entre outros ${ }^{10}$.

Sobre o uso de tabaco, o IBGE e o Ministério da Saúde realizaram uma pesquisa especial de Tabagismo (PETAB) com pessoas de 15 anos ou mais. Essa pesquisa mostrou que em 2008, 17,2\% da população com mais de 15 anos era fumantes - cerca de 24 milhōes. Em 1989, essa taxa de fumantes era de $33,1 \%$. Assim, confirmase a reduçáo do tabagismo no Brasil, o que mostra que as campanhas promovidas nas últimas duas décadas por meio de advertências sanitárias, bem como estratégias de reduçáo do uso do tabaco, vêm obtendo êxito ${ }^{32}$.

Um estudo mostrou que esse comportamento de risco tende a ser mais prevalente entre os estudantes do último ano da área da saúde, os quais deveriam possuir mais conhecimento acerca dos hábitos que favorecessem a promoção da saúde 7 . Porém, a baixa prevalência de estudantes usuários de tabaco e álcool no presente estudo refuta o achado de Franca e Colares e confirma a hipótese de que profissionais da saúde, principalmente de Educação Física, por adquirirem conhecimentos voltados para a qualidade de vida, bem estar, saúde e a importância da prática regular de atividade física e ter a incumbência de servir de exemplo para alunos e pacientes, tendem a apresentar baixa prevalência em hábitos tidos com maléficos à saúde.

Neste estudo, os quesitos dormir bem e sentir-se descansado, ser capaz de lidar com o estresse do dia-adia e relaxar e desfrutar do tempo livre de lazer estiveram diretamente associados ao estilo de vida adequado (p-valor $<0,05$ ).

Os participantes da pesquisa que declaram saber lidar com o estresse do dia a dia tendem a apresentar um melhor nível de estilo de vida. Sobre o estresse, Nahas ${ }^{19}$ afirma que é algo que não deva ser evitado, e sim ser reconhecido como uma ameaça à saúde e controlá-lo na medida que o torne um estímulo necessário para adaptaçóes positivas no nosso organismo. $\mathrm{O}$ autor ainda arrisca dizer que o estresse é o tempero da vida e a sua ausência faz que o indivíduo deixe de se adaptar e restabelecer a harmonia no organismo. Segundo o mesmo autor, a pessoa que não controla o estresse estará sujeito a alteraçóes comportamentais severas e graves, desde crises nervosas, agressividade, frustração, tristeza e sensação de impotência, que, somadas, podem acarretar diversos tipos de doenças, destacando-se as cardíacas, derrames cerebrais, úlceras, depressão, atrites, alergias, enxaquecas, entre outros.

Universitários mais jovens e que estâo cursando o último ano sofrem pressões quanto a insegurança sobre a inserção no mercado de trabalho e a alta competitividade, diminuição do tempo de lazer e as expectativas geradas pela família acabam gerando níveis elevados de estresse ${ }^{11}$. Em alunos de pós-graduação o nível de estresse pode ser ainda maior ${ }^{33}$.

Outro dado importante encontrado no presente estudo foi que $100 \%$ dos investigados que alegaram desfrutar do tempo livre apresentaram um estilo da vida adequado. Nos cursos presenciais é sabido que a rotina de atividades que o acadêmico se envolve é elevada, desde as aulas regulares, o tempo para estudos individualizados ou em grupo, horas dedicadas a projetos e encontros de extensão e pesquisa. Entretanto, estudos mostram que parte desses estudantes conseguem desfrutar do tempo livre, durante o dia, tendo em vista que a grande maioria náo exerce nenhum tipo de trabalho remunerado ${ }^{4,10}$.

No caso dos alunos participantes da pesquisa, dos quais $74,1 \%$ trabalham mais de 8 horas por dia, as atividades acadêmicas dividem espaço com a jornada de trabalho e a vida pessoal. Ainda, a rotina de estudos tende a ser maior que no modelo presencial, ao considerar-se o caráter 
autônomo em um grau mais elevado que o ensino à distância exige. Mesmo assim, 58,2\% reconhecem a importância de dedicar um momento do dia para realizar atividades de lazer. Talvez o fato de serem indivíduos mais velhos quando comparados a populaçóes de estudantes de cursos presencias e, ainda, por apresentarem um nível socioeconômico alto, facilite o aproveitamento do tempo de lazer.

A boa taxa de estudantes que declararam utilizar cinto de segurança e praticar sexo seguro pode estar relacionada à faixa etária avançada $(33,5 \pm 8,3)$ e a maior proporção de casados ou em união estável $(66,1 \%)$. Por tais características subentende-se maior maturidade e reconhecimento dos riscos relacionados ao não uso do cinto de segurança e a prática de sexo sem segurança. Por outro lado, estudantes de cursos presenciais que responderam aos mesmos questionamentos, declararam alta prevalência de condutas de risco relacionadas a esses fatores, principalmente aqueles do final do curso ${ }^{7}$.

O comportamento de pensar de forma positiva e ser otimista, assim como não sentir-se com raiva e hostil ou triste e deprimido, apresentaram forte dependência com o estilo de vida adequado, o que mostra que o controle da raiva e a priorização por pensamentos positivos em relação as expectativas da vida podem proporcionar uma melhora no estilo de vida global em relaçáo aos estudantes que sentem-se frequentemente com raiva e hostil e pensam de forma negativa e pessimista.

$\mathrm{Na}$ fase universitária existe um aumento no interesse por novos conhecimentos e experiências, e é normal a exploração das capacidades pessoais em busca da autonomia, do amor e da amizade. Alunos ingressantes, ao assumirem maiores responsabilidades sociais, familiares e profissionais, tendem a apresentar um nível de otimismo maior que os concludentes ${ }^{30}$.

Como mostra os resultados da análise de dependência entre o estilo de vida global e fato de estar satisfeito com o trabalho, pode-se perceber que aqueles indivíduos que estão satisfeitos com o emprego tendem a possuir um estilo de vida mais adequado.

Atualmente, sabe-se que o desgaste no trabalho é um dos aspectos que pode estar relacionado à ocorrência de agravos à saúde. $\mathrm{O}$ excesso de horas e a intensificação do esforço físico no trabalho, que na maioria dos casos independe da vontade dos profissionais, são fatores considerados fundamentais nas análises sobre a carga de trabalho e suas relaçóes com a fadiga, o burnout, as doenças cardiovasculares e a ocorrência de acidentes.

Entretanto, a população participante da pesquisa está lotada em pequenas cidades do interior do estado do Paraná, que não ultrapassam mais de 50 mil habitantes. Cidades desse porte tendem a apresentar condiçóes de trabalho e transporte menos fatigantes que os grandes centros $^{34}$.

\section{Conclusões}

A identificação dos fatores de risco pode significar, na medida do possível, as circunstâncias ou comportamentos que podem estar conferindo ao indivíduo uma maior probabilidade de desenvolver uma determinada condição de agravo à saúde.

Portanto, apresentar as principais características pessoais dos acadêmicos de Educação Física entrevistados que estão correlacionadas a obtenção de um estilo de vida adequado foi o principal feito desta pesquisa. A socialização, a dieta balanceada, dormir bem, controlar o estresse do dia-a-dia, relaxar e desfrutar do tempo livre, controlar a raiva e a tensão e pensar de forma positiva e otimista, são alguns dos fatores que podem estar contribuindo para a maior probabilidade de possui um estilo de vida adequado.

Ainda, o presente estudo vem contribuir com a identificação do perfil do estilo de vida do universitário brasileiro do ensino à distância, o qual pouco se conhece devido à escassez de estudos com esta população.

Quanto às limitaçóes do estudo, destaca-se que pesquisas que se baseiam em questionários auto respondidos possuem limitaçóes quanto à veracidade das respostas, no caso, podendo haver menores taxas de relatos de comportamentos socialmente condenáveis.

Notou-se que o conhecimento adquirido sobre estilo de vida saudável durante a graduação por si só não é um fator que garante a prática de certos comportamentos saudáveis, principalmente a prática regular de atividade física, já que não existiu relação direta entre esse hábito e o estilo de vida. Estes achados mostram ainda que deve haver uma preocupação contínua das instituições de ensino superior que oferecem cursos na modalidade à distância em conhecer as dimensóes do estilo e da qualidade de vida de seus estudantes.

Maior preocupação ainda deve haver sobre o futuro professor de Educação Física legitimado como profissional da saúde, tendo em vista sua atuaçáo direta e responsabilidade ímpar no processo educativo das crianças e adolescentes. Sua contribuição não se limita apenas em vincular os conteúdos de sua área de ensino, mas transferir conhecimento, dividir experiências e exemplificar valores humanos. Logo, é esperado desse profissional uma conduta de liderança, tendo como pilar fundamental o exemplo.

\section{Referências}

1. Leite T, Santos B. Pressão arterial e estilo de vida de estudantes universitários. Rev Bras Ciên Saúde. 2011;9(27):14-20.

2. World Health Organization. The World Health Report 1998: Life in the 21st century a vision for all. WHO: 1998.

3. Rozmus C, Evans R, Wysochansky M, Mixon D. An 
analysis of health promotion and risk behaviors of freshman college students in a rural southern setting. Pediatr Nurs. 2005;20(1):25-33.

4. Joia L. Perfil do estilo de vida individual entre estudantes universitários. Rev Movimenta. 2010;3(1):16-23.

5. Palma A, Abreu R, Cunha C. Comportamentos de risco e vulnerabilidade entre estudantes de educação física. Rev Bras Epidemiol. 2007;10(1):117-26.

6. Cardoso B, dos Santos M, Berardinelli L. A relação estilo de vida e tabagismo entre acadêmicos de enfermagem. Rev Eletrônica Enferm. 2009;11(2)368-74.

7. Franca C, Colares V. Estudo comparativo de condutas de saúde entre universitários no início e no final do curso. Rev Saúde Públ. 2008;42(3):420-7.

8. Martins G, MR S, Prates M, Martins G. Análise dos parâmetros de qualidade e estilo de vida de universitários. Rev Mackenzie Educ Fís Esporte. 2012;11(1):22-30.

9. Paixão L, Dias R, Prado W. Estilo de vida e estado nutricional de universitários ingressantes em cursos da área de saúde do Recife/PE. RBAFS. 2012;15(3):145-50.

10. Moraes M, Laat E, Lara L, Leite G. Consumo de álcool, fumo e qualidade de vida: um comparativo entre universitários. Cinergis. 2011;11(1):86-94.

11. Santos G, Venâncio S. Perfil do estilo de vida de acadêmicos concluintes em educaçáo física do centro universitário do leste de Minas Gerais UNILESTE-MG. Movimentum. 2006;1:1-18.

12. Silva D. Nível de atividade física e fatores associados em acadêmicos de educaçáo física de uma universidade pública do nordeste do Brasil. RBAFS. 2012;16(3):193-8.

13. Vargas L, Redkva P, Cantorani J, Szesz Junior A, Pilatti L, Gutierrez G. Validação dos questionários eltrônicos IPAQ e Estilo de Vida Fantástico em estudantes universitários de Educação Física na modalide a distância. RBQV. 2013;5:9-18.

14. Pesquisa ABCDE. Critério de classificação Econômica Brasil. São Paulo. 2012. p. 3.

15. Baretta E, Baretta M, Peres K. Nível de atividade física e fatores associados em adultos no Município de Joaçaba, Santa Catarina, Brasil. Cad Saúde Públ. 2007;23(7):1595-602.

16. Añez C, Reis R, Petroski E. Versão brasileira do questionário" estilo de vida fantástico": tradução e validação para adultos jovens. Arq Bras Cardiol. 2008;91(2):102-9.

17. Wilson D, Nielsen E, Ciliska D. Lifestyle assessment: testing the FANTASTIC instrument. Can Fam Physician. 1984;30:1863.

18. Barros MVG, Reis R, Hallal P, Florindo A, Farias Júnior J. Análise de dados em saúde. $3^{\mathrm{a}}$ ed. Londrina: Midiograf; 2012.

19. Nahas M. Atividade física, saúde e qualidade de vida: conceitos e sugestóes para um estilo de vida ativo. $2^{\text {a }}$ ed. Londrina: Midiograf; 2001.
20. Lovisolo H, Votre S, Costa V. Normas, utilidades e gostos na aprendizagem. In: Votre SJ, Costa VLMC, (editores). Cultura, atividade corporal e esporte. Rio de Janeiro: Editora Gama Filho; 1995:213-31.

21. Martins M, Ricarte I, Rocha C, Maia R, Silva V, Veras A, et al. Pressão arterial, excesso de peso e nível de atividade física em estudantes de universidade pública. Arq Bras Cardiol. 2010;95(2):192-9.

22. Reichert F, Barros A, Domingues M, Hallal P. The role of perceived personal barriers to engagement in leisure-time physical activity. Ame J Pub Health. 2007;97:515-9.

23. Mielke G, Ramis T, Habeyche E, Oliz M, Tessmer M, Azevedo $\mathrm{M}$, et al. Atividade física e fatores associados em universitários do primeiro ano da Universidade Federal de Pelotas. RBAFS. 2012;15(1):57-64.

24. Castro Júnior E, Barreto L, Oliveira J, Almeida P, Leite J. Avaliação do nível de atividade física e fatores associados em estudantes de medicina de Fortaleza-CE. Rev Bras Ciênc Esporte. 2012;34(4):955-67.

25. Marcondelli P, Costa T, Schmitz B. Nível de atividade física e hábitos alimentares de universitários do $3^{\circ}$ ao $5^{\circ}$ semestres da área da saúde. Rev Nutr. 2008;21(1):39-47.

26. Simão C, Nahas M, Oliveira E. Atividade física habitual, hábitos alimentares e prevalência de sobrepeso e obesidade em universitários da Universidade do Planalto CatarinenseUNIPLAC, Lages. SC. RBAFS. 2012;11(1):3-12.

27. Bielemann R, Karine G, Azevedo M, Reichert F. Prática de atividade física no lazer entre acadêmicos de Educação Física e fatores associados. RBAFS. 2012;12(3):65-72.

28. Silva G, Bergamaschine R, Rosa M, Melo C, Miranda R, Filho M. Avaliaçẫo do nível de atividade física de estudantes de graduação das áreas saúde/biológica. RBAFS. 2007;11:3-12.

29. Fontes A, Vianna R. Prevalence and factors related to low level physical activity among university students in a public university in the northeast region of Brazil. Rev Bras Epidemiol. 2009;12(1):20-9.

30. Santos J. Estilo de vida relacionado à saúde de estudantes universitários: comparaçáo entre ingressantes e concluintes [tese]. Porto: Universidade Fernando Pessoa; 2011.

31. Vieira D, Ribeiro M, Romano M, Laranjeira R. Álcool e adolescentes: estudo para implementar políticas municipais. Rev Saúde Públ. 2007;41(3):396-403.

32. Insituto Nacional do Cancêr. Pesquisa especial de tabagismo - PETAB: relatório Brasil. Rio de Janeiro: INCA; 2011.

33. Malagris L, Suassuna A, Bezerra D, Hirata H, Monteiro J, Silva L, et al. Níveis de estresse e características sociobiográficas de alunos de pós-graduação. Psicol Rev. 2009;15(2):184-203.

34. Paiva R. Integraçáo de ciclovias com outros modos de transporte. Workshop Internacional sobre Planejamento e Implementação de Sistemas Cicloviários; 2006; Guarulhos. 